\title{
Quality of Recovery After Low-Pressure Laparoscopic Donor Nephrectomy Facilitated by Deep Neuromuscular Blockade: A Randomized Controlled Study
}

\author{
Denise M. D. Özdemir-van Brunschot ${ }^{1}$ - Gert J. Scheffer ${ }^{2} \cdot$ Michel van der Jagt ${ }^{1}$ • \\ Hans Langenhuijsen ${ }^{3}$ - Albert Dahan ${ }^{4}$ - Janneke E. E. A. Mulder ${ }^{2}$. \\ Simone Willems ${ }^{2} \cdot$ Luuk B. Hilbrands $^{5} \cdot$ Rogier Donders $^{6} \cdot$ Cees J. H. M. van Laarhoven ${ }^{1}$. \\ Frank A. d'Ancona ${ }^{3} \cdot$ Michiel C. Warlé ${ }^{1}$
}

Published online: 12 June 2017

(c) The Author(s) 2017. This article is an open access publication

\begin{abstract}
Background The use of low intra-abdominal pressure $(<10 \mathrm{mmHg})$ reduces postoperative pain scores after laparoscopic surgery.

Objective To investigate whether low-pressure pneumoperitoneum with deep neuromuscular blockade improves the quality of recovery after laparoscopic donor nephrectomy (LDN).

Design, setting and participants In a single-center randomized controlled trial, 64 live kidney donors were randomly assigned to 6 or $12 \mathrm{mmHg}$ insufflation pressure. A deep neuromuscular block was used in both groups. Surgical conditions were rated by the five-point Leiden-surgical rating scale (L-SRS), ranging from 5 (optimal) to 1 (extremely poor) conditions. If the L-SRS was insufficient, the pressure was increased stepwise.

Main outcome measure The primary outcome measure was the overall score on the quality of recovery-40 (QOR-40) questionnaire at postoperative day 1.

Results The difference in the QOR-40 scores on day 1 between the low- and standard-pressure group was not significant $(p=.06)$. Also the overall pain scores and analgesic consumption did not differ. Eight procedures $(24 \%)$, initially started with low pressure, were converted to a standard pressure $(\geq 10 \mathrm{mmHg})$. A L-SRS score of 5 was significantly more prevalent in the standard pressure as compared to the low-pressure group at $30 \mathrm{~min}$ after insufflation $(p<.01)$.

Conclusions Low-pressure pneumoperitoneum facilitated by deep neuromuscular blockade during LDN does not reduce postoperative pain scores nor improve the quality of recovery in the early postoperative phase. The question whether the use of deep neuromuscular blockade during laparoscopic surgery reduces postoperative pain scores independent of the intra-abdominal pressure should be pursued in future studies.

Trial registration The trial was registered at clinicaltrial.gov before the start of the trial (NCT02146417).
\end{abstract}

Michiel C. Warlé

Michiel.Warle@radboudumc.nl

1 Department of Surgery, Radboud University Medical Center, Geert Grooteplein-Zuid 10, 6525 GA Nijmegen, The Netherlands

2 Department of Anesthesiology, Radboudumc, Nijmegen, The Netherlands

3 Department of Urology, Radboudumc, Nijmegen, The Netherlands
4 Department of Anesthesiology, Leiden University Medical Centre, Leiden, The Netherlands

5 Department of Nephrology, Radboudumc, Nijmegen, The Netherlands

6 Department of HTA, Radboudumc, Nijmegen, The Netherlands 


$\begin{array}{ll}\text { Abbreviations } \\ \text { CRR2 } & \text { Creatinine reduction ratio on day } 2 \\ \text { DGF } & \text { Delayed graft function } \\ \text { EBL } & \text { Estimated blood loss } \\ \text { LDN } & \text { Laparoscopic donor nephrectomy } \\ \text { ORT } & \text { Operation time } \\ \text { NMB } & \text { Deep neuromuscular block } \\ \text { PNP } & \text { Pneumoperitoneum } \\ \text { PTC } & \text { Post-tetanic count } \\ \text { SGF } & \text { Slow graft function } \\ \text { TOF } & \text { Train-of-four } \\ \text { WIT1 } & \text { First warm ischemia time }\end{array}$

\section{Introduction}

Laparoscopic donor nephrectomy (LDN) has several advantages over open donor nephrectomy, e.g., shorter length of hospital stay, earlier return to normal physical function and reduced use of analgesics [1]. The use of low intra-abdominal insufflation pressure decreases postoperative pain in laparoscopic cholecystectomy [2,3], and also evidence exists that postoperative pain is decreased when low-pressure pneumoperitoneum (PNP) is used during LDN [4]. However, the use of low-pressure PNP can impair surgical field visualization $[5,6]$. To optimize the quality of the surgical conditions, Madsen et al. [7] used a deep neuromuscular block (NMB) to enhance surgical space, measured as the distance from the sacral promontory to the trocar. Furthermore, Dubois and Staehr-Rye showed that the use of a deep neuromuscular block (NMB) improves surgical conditions during laparoscopic hysterectomy and laparoscopic cholecystectomy, respectively $[8,9]$.

In this study, we addressed the hypothesis that the use of low-pressure PNP $(<10 \mathrm{mmHg})$ during laparoscopic donor nephrectomy improves the early quality of recovery as compared to the use of standard-pressure PNP $(\geq 10 \mathrm{mmHg})$. A deep NMB was used to facilitate the use of the low-pressure PNP.

\section{Methods}

\section{Patients}

Sixty-four live kidney donors were recruited between August 2014 and July 2015, and written informed consent was obtained. All adult patients eligible for live kidney donation after multidisciplinary discussion were eligible for this study. Exclusion criteria included: insufficient knowledge of the Dutch language to read the patient information and to fill out the questionnaires, chronic use of analgesics or psychotropic drugs, known or suspect allergy to rocuronium or sugammadex, the presence of neuromuscular disease and the need for rapid sequence induction. The study was approved by the institutional review board, the protocol was published [10] and the study was registered at clinicaltrial.gov (NCT02146417).

\section{Randomization and blinding}

Patients were randomly assigned to two groups: 'lowpressure PNP,' defined as $6 \mathrm{mmHg}$ or 'standard-pressure PNP,' defined as $12 \mathrm{mmHg}$. Since the use of deep NMB may influence early postoperative recovery and other outcome parameters, deep NMB was also used in the control group (standard-pressure PNP). The kidney is often more adhesive in men as compared to women. Also the retrieval of a left kidney usually is more time-consuming due to side branches of the left renal vein. To control for these factors, we stratified for gender and side of donor nephrectomy. Block randomization was performed using a computergenerated randomization code.

All surgeons, anesthesiologists and the research team were blinded. All monitors indicating the intra-abdominal pressure were covered during the procedure. After intubation, a nurse opened a sealed envelope containing the allocation of treatment and subsequently installed the intraabdominal pressure. The same nurse monitored the intraabdominal pressure and performed adjustments when required. To assess whether the blinding procedure sufficed in keeping the primary surgeon ignorant of the treatment allocation, he was asked to guess at the end of the procedure whether low or standard pressure was used.

\section{Anesthesia and surgery}

All patients received intravenous anesthesia with $1-3 \mathrm{mg} /$ $\mathrm{kg}$ propofol and $0.2-0.5 \mu \mathrm{g} / \mathrm{kg}$ sufentanil. Before administration of rocuronium, the TOF-watch (TOF-watch-SX, MSD BV, Oss, the Netherlands) was calibrated. Rocuronium $1 \mathrm{mg} / \mathrm{kg}$ was administered, and the patient was intubated. Anesthesia was maintained by continuous infusion of $0.05-0.5 \mu \mathrm{g} / \mathrm{kg} / \mathrm{h}$ sufentanil, sevoflurane (1 MAC) and rocuronium $0.3 \mathrm{mg} / \mathrm{kg} / \mathrm{h}$. Deep NMB was defined as a post-tetanic count (PTC) of 1-5. All patients received sugammadex $4 \mathrm{mg} / \mathrm{kg}$ after surgery. Patients were extubated when the TOF ratio was at least $90 \%$.

All primary surgeons had performed at least 50 laparoscopic donor nephrectomies. First, a Hasson trocar was introduced and the PNP was established. Subsequently three other trocars were placed under direct vision. After opening of Gerota's fascia, the renal artery, vein and ureter 


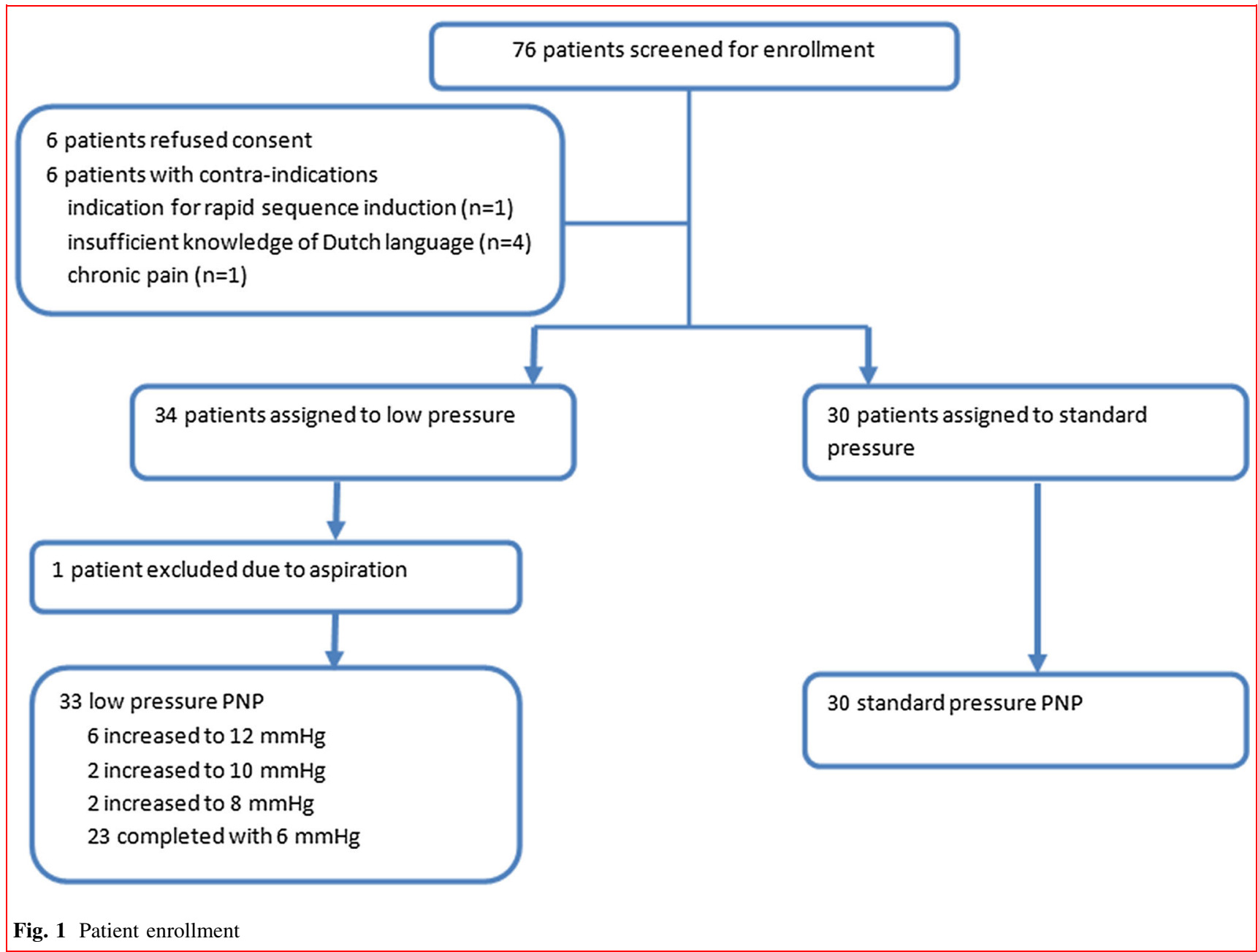

were identified and dissected. When present, the gonadal, suprarenal and/or lumbal vein were clipped and transected. Then, a Pfannenstiel incision was made. The renal artery and vein were transected using an endostapler, and the kidney was extracted using an endobag. The kidney was immediately flushed at the back table.

After surgery, all patients received patient-controlled analgesia (PCA) with intravenous administration of piritramide (bolus $1 \mathrm{mg}$, lock-out $6 \mathrm{~min}$ ) and acetaminophen (4000 mg daily). PCA was stopped at day 2 and was replaced by oxycodon. Patients did not receive local anesthetics.

\section{Evaluation of perioperative conditions}

During the laparoscopic procedure, surgical conditions were measured after introduction of the trocars and then every $15 \mathrm{~min}$. Surgical conditions were evaluated by means of the surgical rating score (SRS), first described by Martini et al. [11]. The SRS ranged from 1 to 5, extremely poor (1), poor (2), adequate (3), good (4) or optimal (5) depending on the subjective judgment of the primary surgeon. When the overall score was $\leq 3$, intra-abdominal pressure was stepwise increased with $2 \mathrm{mmHg}$. In case the pressure was already set at $12 \mathrm{mmHg}$ (control group), the nurse was instructed to pretend increasing the pressure stepwise, while keeping the pressure set at $12 \mathrm{mmHg}$. The study flowchart is shown in Fig. 1.

\section{Outcome measures}

The primary outcome measure was the overall score on the quality of recovery (QoR-40) questionnaire on the first operative day. Secondary outcome measures included: perioperative parameters (PNP) duration, operation time (ORT), first warm ischemia time (WIT1), estimated blood loss (EBL), intra- and postoperative complications and postoperative pain scores. Blood loss was recorded by estimating the amount of blood $(\mathrm{ml})$ in the collection bottle of the suction device after skin closure. Postoperative complications were recorded during the first postoperative days and graded according to the Clavien-Dindo 
Table 1 Patient characteristics

\begin{tabular}{llll}
\hline & Low pressure $(n=33)$ & Standard pressure $(n=30)$ & $p$ value \\
\hline Age (year) & $54.1($ SD 13.2) & $55.9($ SD 10.7) & .57 \\
Male gender & $19(55.9 \%)$ & $17(56.7 \%)$ & .95 \\
BMI $\left(\mathrm{kg} / \mathrm{m}^{2}\right)$ & $25.5($ SD 3.2) & $26.4($ SD 3.1) & .31 \\
Preoperative serum creatinine $(\mu \mathrm{mol} / \mathrm{l})$ & $74.4($ SD 13.1) & $75.0($ SD 11.9) & .84 \\
\hline
\end{tabular}

$B M I$ body mass index

classification. Overall, superficial wound, deep abdominal and referred shoulder pain scores (in rest and upon movement) were recorded as numeric rating scores, using a 11-point Likert scale ranging from 0 to 10 [4]. Superficial wound pain was defined as sharp pain located in the area of the incision(s), deep abdominal pain as a dull and more diffuse pain in the abdomen and referred shoulder pain as pain in the shoulder area.

Also, graft function of the recipient was evaluated. Delayed graft function (DGF) was defined as the need for dialysis in the first postoperative week, excluding when needed for hyperkalemia [12]. Slow graft function (SGF) was defined as serum creatinine $>3.0 \mathrm{mg} / \mathrm{dl}$ at day 5 , without the need for dialysis [13].

\section{Sample size calculation and data analysis}

A ten-point difference in the overall score in the QoR-40 questionnaire on day 1 was considered a minimal clinically relevant difference [14-16]. Based on previous studies, we used a standard deviation (SD) for the QOR-40 score at day one of 14 [14-20]. A sample size of 32 patients per group was required to provide $80 \%$ power.

Data were analyzed on an intention-to-treat basis. To control for covariates, i.e., age, gender and side of donor nephrectomy, multivariable logistic regression was used. All analyses were performed using SPSS version 22 (SPSS Inc., Chicago, IL, USA). $p$ values $<.05$ were considered significant.

\section{Results}

\section{Patient characteristics}

A total of 76 patients were screened for enrollment, six patients refused informed consent and six patients met one of the exclusion criteria. A total of 34 patients were allocated to the low-pressure PNP group and 30 to the standard-pressure PNP group. For one patient, surgery was canceled due to persistently low oxygen saturation presumably caused by aspiration after induction. According to the protocol, this patient was not replaced. Patient demographics are shown in Table 1, and there were no significant differences in baseline characteristics.

\section{Primary outcome measure}

Mean QoR-40 score on day 1 was 171.2 (SD 14.5) in the low-pressure group versus 165.4 (SD 14.6) in the standardpressure group $(p=.12$ ), as shown in Table 2. After correction of age and gender, there was no significant difference in QoR-40 score on day 1 (adjusted $p=.06$ ). Perprotocol analysis showed a mean QoR-40 score on day 1 of 170.5 (SD 15.6) in the low-pressure group versus 166.9 (SD 14.0) in the standard-pressure group $(p=.35)$.

\section{Secondary outcome measures}

Separate analyses of the dimensions of the QoR-40 questionnaire showed that patients allocated to the low-pressure group had significantly higher scores regarding physical support at day 1 (adjusted $p=.01$ ) and emotional status and physical independence at day 2 (adjusted $p$ values are .03 and $<.01$, respectively), see Table 2 . Surgical parameters are shown in Table 3. Mean ORT was 7.8 min longer for low-pressure LDN, which was mainly due to a longer PNP phase. EBL was significantly higher for the lowpressure group, respectively, $48.3 \mathrm{ml}$ (SD 66) versus $22.7 \mathrm{ml}$ (SD 25.4). There were no significant differences in WIT1, conversion to HALDN, or intra-operative complications. With regard to overall pain scores and analgesic consumption, no significant differences were observed between the low- and standard-pressure PNP group, as shown in Table 4. The deep intra-abdominal pain component was significantly lower at postoperative day 2 in patients allocated to the low-pressure group, respectively, 0.8 (SD 1.1) versus 1.8 (SD 2.3).

\section{Surgical conditions and complications}

During the procedure, it was necessary to increase the intra-abdominal pressure to $8 \mathrm{mmHg}$ in two patients, to $10 \mathrm{mmHg}$ in two patients and to $12 \mathrm{mmHg}$ in six patients. In Fig. 2, it is shown that a SRS score of 5 (optimal conditions) was significantly more prevalent in the standard 
Table 2 QoR-40 questionnaire

\begin{tabular}{|c|c|c|c|c|c|}
\hline Intention-to-treat & Low pressure $(n=33)$ & Standard pressure $(n=30)$ & \multicolumn{2}{|c|}{$p$ value } & Adjusted $p$ value* \\
\hline \multicolumn{6}{|l|}{ Overall score } \\
\hline Preoperative & 198.9 (SD 1.7) & $198.5(\mathrm{SD} 2.6)$ & .46 & & .43 \\
\hline Postoperative day $1^{\#}$ & $171.2(\mathrm{SD} 14.5)$ & $165.4(\mathrm{SD} 14.6)$ & .12 & & .06 \\
\hline Postoperative day 2 & $185.6(\mathrm{SD} 15.3)$ & $179.8($ SD 20.4) & .21 & & .14 \\
\hline Postoperative day 7 & $186.2(\mathrm{SD} 12.2)$ & $186.0(\mathrm{SD} 11.5)$ & .94 & & .92 \\
\hline \multicolumn{6}{|l|}{ Physical comfort } \\
\hline Preoperative & $59.3(\mathrm{SD} 1.4)$ & $59.4(\mathrm{SD} 1.5)$ & .90 & & .99 \\
\hline Postoperative day 1 & 53.2 (SD 5.9) & $52.2(\mathrm{SD} 8.8)$ & .61 & & .41 \\
\hline Postoperative day 2 & $53.8(\mathrm{SD} 6.8)$ & $52.0(\mathrm{SD} \mathrm{12})$ & .88 & & .39 \\
\hline Postoperative day 7 & $55.9(\mathrm{SD} 5.2)$ & $56.6(\mathrm{SD} 3.4)$ & .57 & & .56 \\
\hline \multicolumn{6}{|l|}{ Emotional status } \\
\hline Preoperative & 49.7 (SD 0.7) & $49.3($ SD 1.1) & .11 & & .10 \\
\hline Postoperative day 1 & $46.1(\mathrm{SD} 4.0)$ & $46.4(\mathrm{SD} 3.9)$ & .82 & & .98 \\
\hline Postoperative day 2 & 48.4 (SD 5.3) & 46.3 (SD 3.9) & .08 & & .03 \\
\hline Postoperative day 7 & $47.3(\mathrm{SD} 3.6)$ & $46.9(\mathrm{SD} 4.0)$ & .70 & & .71 \\
\hline \multicolumn{6}{|l|}{ Physical independence } \\
\hline Preoperative & $25.0(\mathrm{SD} 0.2)$ & $24.9(\mathrm{SD} 0.4)$ & .61 & & .64 \\
\hline Postoperative day 1 & $22.7(\mathrm{SD} 7.0)$ & $21.2(\mathrm{SD} 3.7)$ & .31 & & .28 \\
\hline Postoperative day 2 & $21.3(\mathrm{SD} 1.6)$ & $19.7(\mathrm{SD} 3.1)$ & .01 & & .00 \\
\hline Postoperative day 7 & $22.6(\mathrm{SD} 1.6)$ & $22.4($ SD 1.7) & .67 & & .64 \\
\hline \multicolumn{6}{|l|}{ Support } \\
\hline Preoperative & $30.0($ SD 0.0$)$ & $30.0(\mathrm{SD} .2)$ & .33 & & .29 \\
\hline Postoperative day 1 & 21.9 (SD 3.4) & 19.9 (SD 2.3) & .01 & & .01 \\
\hline Postoperative day 2 & $29.8($ SD 0.6) & $29.5(\mathrm{SD} 1.4)$ & .21 & & .16 \\
\hline Postoperative day 7 & $28.5($ SD 3.3) & $29.2($ SD 2.0) & .31 & & .36 \\
\hline \multicolumn{6}{|l|}{ Pain } \\
\hline Preoperative & 34.9 (SD 0.3) & 34.9 (SD .4) & .93 & & .86 \\
\hline Postoperative day 1 & $31.1(\mathrm{SD} 3.3)$ & $29.5(\mathrm{SD} 4.2)$ & .12 & & .08 \\
\hline Postoperative day 2 & $32.3(\mathrm{SD} 4.9)$ & $32.4(\mathrm{SD} 6.1)$ & .99 & & .99 \\
\hline Postoperative day 7 & 31.9 (SD 2.6) & 30.9 (SD 3.4) & .19 & & .17 \\
\hline Per-protocol & Low pressure $\leq 10 \mathrm{mmHg}^{\S}(n=25)$ & Standard pressure $>10 \mathrm{mmH}$ & & $p$ value & Adjusted $p$ value* \\
\hline \multicolumn{6}{|l|}{ Overall score } \\
\hline Preoperative & 198.7 (SD 1.8) & 198.7 (SD 2.5) & & .95 & .94 \\
\hline Postoperative day 1 & $170.5(\mathrm{SD} 15.6)$ & $166.9(\mathrm{SD} 14.0)$ & & .35 & .09 \\
\hline Postoperative day 2 & 185.6 (SD 11.3) & 180.7 (SD 21.7) & & .30 & .12 \\
\hline
\end{tabular}

Significant $p$ values are given in bold

QoR40 quality of recovery-40 score

\# Primary study endpoint

* $p$ value adjusted for age and gender

$\S$ For the per-protocol analysis, patients were considered 'low pressure' if the intra-abdominal pressure maintained $<10 \mathrm{mmHg}$ during the entire procedure

pressure as compared to the low-pressure group at $30 \mathrm{~min}$ after insufflation $(p<.01)$.

Intra- and postoperative complications are shown in Table 5. Two splenic lesions occurred. In one patient, a bladder injury occurred after introduction of the endobag. This complication occurred at a time point where the insufflation pressure was increased to $10 \mathrm{mmHg}$. The lesion was immediately sewed and the urinary catheter remained 5 days in situ where after the patient was discharged without any further consequences. In another patient, the pressure was increased to $20 \mathrm{mmHg}$ to treat persistent venous oozing. This patient was originally 
Table 3 Surgical parameters

\begin{tabular}{|c|c|c|c|}
\hline & Low pressure $(n=33)$ & Standard pressure $(n=30)$ & $p$ value \\
\hline Left kidneys & $30(88.2 \%)$ & $26(86.7 \%)$ & .85 \\
\hline ORT $(\min )$ & $109.4($ SD 27.2) & $101.6($ SD 23.7) & .23 \\
\hline PNP time (min) & $91.6(\mathrm{SD} 30.8)$ & $82.8(\mathrm{SD} 24.9)$ & .22 \\
\hline \multicolumn{4}{|l|}{ Increase in pressure } \\
\hline $8 \mathrm{mmHg}$ & 2 & 0 & \\
\hline $10 \mathrm{mmHg}$ & 2 & 0 & \\
\hline $12 \mathrm{mmHg}$ & 6 & 0 & \\
\hline Conversion to HALDN & $1(3.0 \%)$ & $1(3.3 \%)$ & .95 \\
\hline WIT1 $(\mathrm{sec})$ & $190.0(\mathrm{SD} 60.8)$ & 199.6 (SD 69.2) & .56 \\
\hline EBL (ml) & 48.3 (SD 66.4) & $22.7(\mathrm{SD} 25.4)$ & .05 \\
\hline
\end{tabular}

EBL estimated blood loss, HALDN hand-assisted laparoscopic donor nephrectomy, ORT operation time, PNP pneumoperitoneum and WIT1 first warm ischemia time

allocated to the low-pressure group, but at the time of the bleeding the pressure was already increased to $12 \mathrm{mmHg}$.

For all except two patients, $4 \mathrm{mg} / \mathrm{kg}$ sugammadex was sufficient for reversal of deep NMB. In one patient, an additional dose of $2 \mathrm{mg} / \mathrm{kg}$ sugammadex was administered. In another, obese patient (100 kg body weight) TOF could not be adequately monitored, possibly due to electrode malpositioning. Therefore, a higher rocuronium dose (12 $\mathrm{mg} / \mathrm{kg}$ ) was administered than actually required.

No significant differences were observed in the length of hospital stay between the low- and standard-pressure group, respectively, 2.8 and 3.2 days (Table 5).

The primary surgeons guessed the initial insufflation pressure at the end of the procedure. In 52 of $63(82.5 \%)$ cases, the surgeon guessed the allocation of treatment correctly.

\section{Recipient outcome}

One recipient died because of an ischemic cerebrovascular accident six weeks after transplantation. With regard to recipient kidney graft function, there were no significant differences in postoperative serum creatinine, or the incidence of DGF or SGF (data not shown).

\section{Discussion}

In this study, patients allocated to the low-pressure PNP group did not show a significantly better quality of recovery at postoperative day 1 which was the primary endpoint of this study. However, patients in the low-pressure group needed less physical support at day 1, and their emotional status and physical independence were significantly better at day 2. The QoR-40 questionnaire is a wellvalidated, patient-reported outcome measure regarding five dimensions of the quality of recovery after surgery [17]. Nevertheless, the clinical relevance of an improved score in one or more separate dimensions is unclear.

Pain after laparoscopic surgery can be divided into three components: incisional pain, deep intra-abdominal pain and referred shoulder pain [21]. Although the deep intraabdominal pain score at postoperative day 2 was significantly lower in the low-pressure group, the use of lowpressure PNP did not lead to lower overall pain scores. This is not in accordance with our previous pilot study [4], nor with our recently performed systematic review with metaanalysis comparing pain scores for various laparoscopic procedures [3]. These studies showed significantly lower overall and referred shoulder pain scores in favor of lowpressure PNP. A possible explanation for this discrepancy is that we used a deep NMB in both arms of the study. It has been postulated that a deep NMB more effectively relaxes the abdominal wall musculature as compared to a standard NMB [22]. Therefore, the use of a deep NMB alone (with standard pressure) may reduce pressure-related postoperative pain.

Lindekaer et al. [23] showed that a deep NMB allows a higher intra-abdominal volume with the same intra-abdominal insufflation pressure. To our knowledge, our trial is the first comparing low- versus standard-pressure PNP with the use of deep NMB in both groups. Despite the conversion from low $(6 \mathrm{mmHg})$ to standard pressure $(\geq 10 \mathrm{mmHg}$ ) in eight cases $(24 \%)$, the rating of surgical conditions was significantly better for standard-pressure PNP. Nevertheless, the skin-to-skin operation time was comparable for both groups. More importantly, there was no relevant difference in the intra- and postoperative complication rate between the low- and standard-pressure group. The most important intra-operative complication was an iatrogenic bladder injury in a patient allocated to the low-pressure group. However, this complication 
Table 4 Overall and components of pain scores and analgesic consumption

\begin{tabular}{|c|c|c|c|}
\hline & Low pressure $(n=33)$ & Standard pressure $(n=30)$ & $p$ value \\
\hline \multicolumn{4}{|l|}{ Overall maximum pain score ${ }^{\#}$} \\
\hline Postoperative $1 \mathrm{~h}$ & $4.0(2.0)$ & $4.1(2.5)$ & .84 \\
\hline Postoperative day 1 & $4.7(2.3)$ & $4.9(2.4)$ & .75 \\
\hline Postoperative day 2 & $3.7(2.3)$ & $4.0(2.4)$ & .54 \\
\hline \multicolumn{4}{|l|}{ Superficial wound component } \\
\hline Postoperative $1 \mathrm{~h}$ & $1.8(2.1)$ & $1.7(2.2)$ & .78 \\
\hline Postoperative $1 \mathrm{~h}$ (movement) & $2.4(2.4)$ & $2.7(2.8)$ & .64 \\
\hline Postoperative day 1 & $1.1(1.6)$ & $0.7(1.4)$ & .28 \\
\hline Postoperative day 1 (movement) & $4.0(2.5)$ & $3.9(2.7)$ & .86 \\
\hline Postoperative day 2 & $0.6(1.0)$ & $0.7(1.3)$ & .67 \\
\hline Postoperative day 2 (movement) & $2.1(1.7)$ & $2.6(2.2)$ & .34 \\
\hline \multicolumn{4}{|l|}{ Deep intra-abdominal component } \\
\hline Postoperative $1 \mathrm{~h}$ & $2.5(1.9)$ & $2.3(2.3)$ & .75 \\
\hline Postoperative $1 \mathrm{~h}$ (movement) & $2.5(2.4)$ & $2.2(2.3)$ & .64 \\
\hline Postoperative day 1 & $1.2(1.8)$ & $2.1(2.1)$ & .09 \\
\hline Postoperative day 1 (movement) & $2.7(2.6)$ & $3.3(2.6)$ & .33 \\
\hline Postoperative day 2 & $0.8(1.1)$ & $1.8(2.3)$ & .02 \\
\hline Postoperative day 2 (movement) & $2.0(2.1)$ & $2.7(2.4)$ & .18 \\
\hline \multicolumn{4}{|l|}{ Referred shoulder component } \\
\hline Postoperative $1 \mathrm{~h}$ & $0.3(1.0)$ & $0.4(1.5)$ & .79 \\
\hline Postoperative $1 \mathrm{~h}$ (movement) & $0.4(1.2)$ & $0.8(2.1)$ & .34 \\
\hline Postoperative day 1 & $1.3(1.9)$ & $1.5(2.3)$ & .78 \\
\hline Postoperative day 1 (movement) & $1.7(2.4)$ & $1.8(2.5)$ & .86 \\
\hline Postoperative day 2 & $1.6(1.7)$ & $1.4(2.2)$ & .60 \\
\hline Postoperative day 2 (movement) & $2.6(2.2)$ & $1.8(2.2)$ & .16 \\
\hline \multicolumn{4}{|l|}{ Analgesic medications } \\
\hline Acetaminophen day $0(\mathrm{mg})$ & $4000(0)$ & $4000(0)$ & 1.0 \\
\hline Acetaminophen day 1 & $4000(0)$ & $4000(0)$ & 1.0 \\
\hline Acetaminophen day 2 & $3895(457)$ & $4000(0)$ & .27 \\
\hline Piritramide day 0 (mg) & $94.2(101.4)$ & 79.9 (114) & .61 \\
\hline Piritramide day 1 & $19.3(18.3)$ & $15.7(14.2)$ & .63 \\
\hline Piritramide day 2 & $0(0)$ & $0(0)$ & 1.0 \\
\hline Oxycodon day 0 (mg) & $0(0)$ & $0(0)$ & 1.0 \\
\hline Oxycodon day 1 & $12.9(13.6)$ & $14.1(6.6)$ & .79 \\
\hline Oxycodon day 2 & $5.3(9.9)$ & $4.8(5.8)$ & .33 \\
\hline
\end{tabular}

\# Maximum score: in rest or after movement including all components of pain (superficial, deep intra-abdominal and referred shoulder pain)

occurred at the end of the procedure, while the intra-abdominal pressure was already increased to a standard pressure $(10 \mathrm{mmHg})$ in an early stage. Therefore, it is unlikely to assume that the bladder injury was related to the use of low-pressure PNP.

The main strength of this study is related to its design as a randomized controlled trial. Live kidney donors in general are healthy individuals and therefore provide a highly homogeneous study population. This reduces the risk of confounding bias. To control for factors that may interfere with the outcome measures, we stratified for gender and side of nephrectomy. Although a slight imbalance occurred during block randomization, which resulted in unequal patient numbers in each group, there were no significant differences in baseline characteristics. Another strength of this study is that the study protocol was published beforehand and that we adhered to the study protocol.

A limitation of this study is that eight patients were converted to a standard pressure $(\geq 10 \mathrm{mmHg}$ ). Therefore, only 25 patients underwent a 'true' low-pressure $(<10 \mathrm{mmHg})$ procedure. This may have blurred the effect on the primary endpoint in the intention-to-treat analysis. 


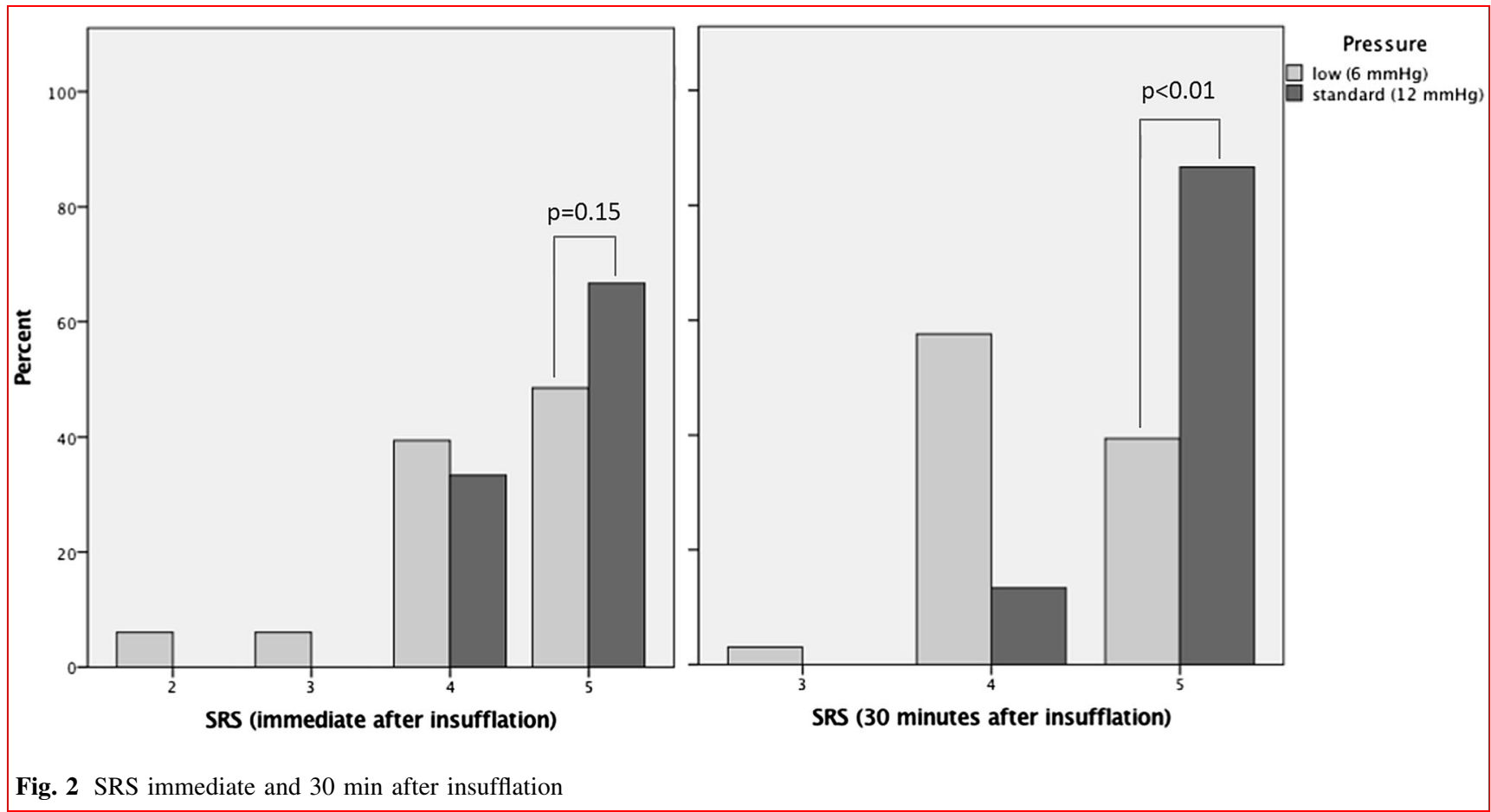

Table 5 Length of hospital stay and complications

\begin{tabular}{|c|c|c|c|}
\hline & $\begin{array}{l}\text { Low pressure } \\
(n=33)\end{array}$ & $\begin{array}{l}\text { Standard pressure } \\
(n=30)\end{array}$ & $p$ value \\
\hline Length of stay (days) & $2.8(\mathrm{SD} 1.1)$ & $3.2(\mathrm{SD} 1.1)$ & .17 \\
\hline \multicolumn{4}{|c|}{ Intra-operative complications } \\
\hline Aspiration & 1 & 0 & \\
\hline Splenic lesion & 1 & 1 & \\
\hline Bladder injury & 1 & 0 & \\
\hline $\begin{array}{l}\text { Additional dose of } \\
\text { sugammadex }\end{array}$ & 0 & 1 & \\
\hline \multicolumn{4}{|c|}{ Postoperative complications } \\
\hline $\begin{array}{l}\text { Fever of unknown } \\
\text { origin }^{\#}\end{array}$ & 0 & 2 & \\
\hline $\begin{array}{l}\text { Urinary tract } \\
\text { infection }^{\S}\end{array}$ & 1 & 0 & \\
\hline Pneumonia $^{\S}$ & 0 & 1 & \\
\hline Gastroparesis $^{\S}$ & 0 & 1 & \\
\hline Total complications & 4 & 6 & .49 \\
\hline
\end{tabular}

\# Clavien-Dindo grade 1 postoperative complication

$\S$ Clavien-Dindo grade 2 postoperative complications

The unexpected high rate of conversions to a standard intra-abdominal pressure may be explained by a learning curve for working with lower pressures. Although all patients were operated by experienced laparoscopic surgeons, it cannot be ruled out that less conversions to a standard pressure would have been required if surgeons had more experience with low-pressure conditions during laparoscopy. Our study protocol did not define a per- protocol analysis. However, a post hoc per-protocol analysis also did not reveal a significant difference with regard to the primary outcome measure (Table 2). Another limitation of the study is the fact that the surgeon could not be fully blinded for the use of low-pressure PNP. In this study, the primary surgeons guessed the initial insufflation pressure, and in $82.5 \%$ of the cases the surgeon guessed the allocation of treatment correctly. In our view, there is no alternative to overcome this limitation. However, it is important to note that the patients were adequately blinded and that a blinded physician assessed all outcome measures. Although the clinically significant difference of the QoR-40 questionnaire is debatable, several studies with comparable types of surgery have used ten points as a clinically significant difference $[14,16]$. After finishing this study, the minimal clinically important difference of the QoR-40 questionnaire was found to be 6.3 in a study by Myles et al. [24]. In our study, the differences in the QoR40 score at postoperative day 1 between low- and standardpressure group after intention-to-treat and per-protocol analyses were 5.8 and 3.6 , respectively. As these differences are smaller than the minimal clinically important difference, it seems unlikely that a larger sample size would lead to different conclusions.

In conclusion, the use of low-pressure pneumoperitoneum with deep NMB did not reduce postoperative pain scores or improve the overall quality of recovery after LDN. As a deep neuromuscular block was also applied in patients allocated to the standard-pressure group, the questions arise whether deep NMB reduces intra- 
abdominal pressure-related pain independent of the intraabdominal pressure. This issue should be addressed in future studies.

Acknowledgements The authors thank M. Ergün, D. van Rijckevorsel and S. Alken for their assistance in data collection.

Funding This study was partly sponsored by Merck \& Co (MSD BV), Haarlem, the Netherlands.

Authors' Contributions DÖ contributed to study design, data collection and analysis and manuscript writing. GS, AD, JM, RD, LH and $\mathrm{CvL}$ contributed to design of the study. FdA contributed to study design and data collection. MvdJ, HL contributed to data collection. MW contributed to study concept and design, data collection and analysis and critical revision of the manuscript. All authors read and approved the final manuscript.

Open Access This article is distributed under the terms of the Creative Commons Attribution 4.0 International License (http://crea tivecommons.org/licenses/by/4.0/), which permits unrestricted use, distribution, and reproduction in any medium, provided you give appropriate credit to the original author(s) and the source, provide a link to the Creative Commons license, and indicate if changes were made.

\section{References}

1. Wilson CH, Sanni A, Rix DA, Soomro NA (2011) Laparoscopic versus open nephrectomy for live kidney donors. Cochrane Database Syst Rev 11:CD006124

2. Gurusamy KS, Samraj K, Davidson BR (2009) Low pressure versus standard pressure pneumoperitoneum in laparoscopic cholecystectomy. Cochrane Database Syst Rev 2:CD006930

3. Ozdemir-van Brunschot DM, van Laarhoven CJ, Scheffer GJ, Pouwels S, Wever KE, Warlé MC (2015) What is the evidence for the use of low-pressure pneumoperitoneum? A systematic review. Surg Endosc 30:2049-2065

4. Warle MC, Berkers AW, Langenhuijsen JF et al (2013) Lowpressure pneumoperitoneum during laparoscopic donor nephrectomy to optimize live donors' comfort. Clin Transplant 27(4): E478-E483

5. Angioli R, Terranova C, Plott F et al (2015) Influence of pneumoperitoneum pressure on surgical field during robotic and laparoscopic surgery: a comparative study. Arch Gynecol Obstet 291(4):865-868

6. Vijayaraghavan N, Sistla SC, Kundra P et al (2014) Comparison of standard-pressure and low-pressure pneumoperitoneum in laparoscopic cholecystectomy: a double blinded randomized controlled study. Surg Laparosc Endosc Percutan Tech 24(2): 127-133

7. Madsen MV, Gatke MR, Sprinborg HH, Rosenberg J, Lund J, Istre O (2015) Optimising abdominal space with deep neuromuscular blockade in gynaecologic laparoscopy-a randomised, blinded crossover study. Acta Anaesthesiol Scand 59(4):441-447

8. Staehr-Rye AK, Rasmussen LS, Rosenberg J et al (2014) Surgical space conditions during low-pressure laparoscopic cholecystectomy with deep versus moderate neuromuscular blockade: a randomized clinical study. Anesth Analg 119(5):1084-1092
9. Dubois PE, Putz L, Jamart J, Marotta ML, Gourdin M, Donnez O (2014) Deep neuromuscular block improves surgical conditions during laparoscopic hysterectomy: a randomised controlled trial. Eur J Anaesthesiol 31(8):430-436

10. Ozdemir-van Brunschot DM, Scheffer GJ, Dahan A et al (2015) Comparison of the effectiveness of low pressure pneumoperitoneum with profound muscle relaxation during laparoscopic donor nephrectomy to optimize the quality of recovery during the early post-operative phase: study protocol for a randomized controlled clinical trial. Trials 16(1):345

11. Martini CH, Boon M, Bevers RF, Aarts LP, Dahan A (2014) Evaluation of surgical conditions during laparoscopic surgery in patients with moderate vs deep neuromuscular block. Br J Anaesth 112(3):498-505

12. Yarlagadda SG, Coca SG, Garg AX et al (2008) Marked variation in the definition and diagnosis of delayed graft function: a systematic review. Nephrol Dial Transplant 23(9):2995-3003

13. Nel D, Vogel J, Muller E, Barday Z, Kahn D (2012) Slow early graft function: a neglected entity after renal transplantation. Nephron Clin Pract 120(4):200-204

14. De Oliviera GS, Fitzgerald PC, Marcus RJ, Ahmad S, McCarthy RJ (2011) A dose-ranging study of the effect of transversus abdominis block on postoperative quality of recovery and analgesia after outpatient laparoscopy. Anesth Analg 113(5):12181225

15. Fitzgerald GS Jr, Ahmad S, Kim J, Rahangdale R, McCarthy R (2014) Transversus abdominis plane infiltration for laparoscopic gastric banding: a pilot study. World J Gastrointest Surg 6(2): $27-32$

16. Rahangdale R, Kendall MC, McCarthy RJ et al (2014) The effects of perineural versus intravenous dexamethasone on sciatic nerve blockade outcomes: a randomized, double-blind, placebocontrolled study. Anesth Analg 118(5):1113-1119

17. Myles PS, Weitkamp B, Jones K, Melick J, Hensen S (2000) Validity and reliability of a postoperative quality of recovery score: the QoR-40. Br J Anaesth 84(1):11-15

18. Kluivers KB, Hendriks CJ, Mol BW et al (2008) Clinimetric properties of 3 instruments measuring postoperative recovery in a gynaecologic surgical population. Surgery 144(1):12-21

19. McIntosh S, Adams J (2011) Anxiety and quality of recobery in day surgery: a questionnaire study using Hospital Anxiety and Depression scale and Quality of Recovery Score. Int J Nurs Pract 17(1):85-92

20. Leslie K, Troedel S, Irwin K et al (2003) Quality of recovery from anesthesia in neurosurgical patients. Anesthesiology 99(5): $85-92$

21. Ergun M, Berkers AW, van der Jagt MF et al (2014) Components of pain assessment after laparoscopic donor nephrectomy. Acta Anaesthesiol Scand 58(2):219-222

22. Kirov K, Motamed C, Combes X, Duvaldestin P, Dhonneur G (2000) Sensibility to atracurium of the lateral abdominal muscles. Ann Fr Anesth Réanim 19:734-738

23. Lindekaer AL, Halvor Springborg H, Istre O (2013) Deep neuromuscular blockade leads to a larger intraabdominal volume during laparoscopy. J Vis Exp 76:e50045

24. Myles PS, Myles DB, Galagher W, Chew C, MacDonald N, Dennis A (2016) Minimal clinically important difference for three quality of recovery scales. Anesthesiology 125(1):39-45 